\title{
Systematic review and meta-analysis of hospital acquired infections rate in a middle east country (1995-2020)
}

\author{
Mohammad Khammarnia ${ }^{1}$, Alireza Ansari-Moghaddam ${ }^{1}$, Eshagh Barfar ${ }^{1}$, Hossein Ansari ${ }^{1}$, Azar Abolpour ${ }^{2}$, \\ Fatemeh Setoodehzadeh $^{1} *$ (D) Javad Shahmohammadi ${ }^{3}$
}

Received: 21 Jun 2020

Published: 10 Aug 2021

\section{Abstract}

Background: Hospital-acquired infections (HAIs) are a global problem in hospitals and significant causes of mortality and morbidity regardless of advances in supportive care, antimicrobial therapy and prevention. The study aimed to determine a comprehensive estimate of the HAIs prevalence, influential factors, and types of these infections in Iran.

Methods: A systematic literature review was conducted according to the PRISMA (Preferred Reporting Items for Systematic Reviews and Meta-Analyses) guidelines using the online databases; Medline, EMBASE, Scopus, Cochrane, SID, Magiran, and Medlib from January 1995 to September 2020 using a combination of medical subject heading terms ("Nosocomial infection [Mesh] OR "' Hospital infection [Mesh] OR Hospital Acquired Infection[Mesh] OR Healthcare-associated infection 'AND ('Iran' [Mesh]) among observational and interventional studies. SPSS version 25 and STATA version 11 were used for data analysis.

Results: A total of 66 (cross-sectional, cohort, and case-control) observational studies were identified. More of the studies had been done before 2014(43 papers or 65\%). Based on the random-effects model, the overall prevalence of HAIs in Iran was 0.111 [95\% CI: $0.105-0.116]$ with a high, statistically significant heterogeneity $(\mathrm{I} 2=99.9 \%)$. The infection rate was 0.157 and 0.089 before and after the Iranian Health Transformation Plan (HTP), respectively. HAIs rates reported more in the South and West of Iran rather than other regions $(0.231$ and 0.164$)(\mathrm{p}=0.001)$. Escherichia coli and klebsiella infections were reported in 53 and 52 papers $(0.239$ and 0.180 , respectively). In addition, respiratory and urinary infections were reported 0.296 and 0.286 in 51 and 38 papers, respectively.

Conclusion: The prevalence of HAIs in Iran is relatively high. Preventing and decreasing hospital nosocomial infections can considerably affect reducing mortality and health-related costs. This should be taken into consideration by health policymakers for pathology and revision of some previous programs and standards as well as the development of appropriate and evidence-based control and education programs to reduce this health problem.

Keywords: Hospital infection, Nosocomial infection, Meta-analysis, Hospital, Iran

Conflicts of Interest: None declared

Funding: None

*This work has been published under CC BY-NC-SA 1.0 license.

Copyright $₫$ Iran University of Medical Sciences

Cite this article as: Khammarnia M, Ansari-Moghaddam A, Barfar E, Ansari H, Abolpour A, Setoodehzadeh F, Shahmohammadi J. Systematic review and meta-analysis of hospital acquired infections rate in a middle east country (1995-2020). Med J Islam Repub Iran. 2021 (10 Aug);35:102. https://doi.org/10.47176/mjiri.35.102

\section{Introduction}

Hospitals are the most important and costly components

Corresponding author: Dr Fatemeh Setoodehzadeh, f.setoodehzadeh@zaums.ac.ir

${ }^{1 .}$ Health Promotion Research Center, Zahedan University of Medical Sciences, Zahedan, Iran

2. School of Health, Student Research Committee, Zahedan University of Medical Sciences, Zahedan, Iran

3. School of Management and Medical Informatics, Shiraz University of Medical Sciences, Shiraz, Iran of health care systems. They account for more than two-

\section{$\uparrow$ What is "already known" in this topic:}

Hospital-acquired infections (HAIs) represent a serious public health concern worldwide. Increased prevalence of HAIs in some cases leads to patient's arbitrary use of drugs, causing severe health hazards as well as significant problems such as drug resistance and death in patients.

\section{$\rightarrow$ What this article adds:}

This study aimed to update and measure the prevalence of HAIs in Iran using a meta-analytic approach. The overall prevalence of HAIs in Iran was $11.1 \%$. The prevalence of HAIs in the South and West of Iran is high. HAIs decreased after Health Transformation Plan in Iran. 
thirds of health care spending. Therefore, they significantly affect the overall health care quality (1). Prevention of infections is part of efforts to improv e the quality of health care services that are vital to patient safety. Hospital-acquired infections (HAIs), also known as nosocomial infections (NI), remain significant causes of mortality and morbidity regardless of advances in supportive care, antimicrobial therapy and prevention (2).

HAIs are a global problem in hospitals (3). The popular definition of hospital infection is an infection that happens within 48 hours after hospitalization, or three days after discharge, or 30 days after surgery. Therefore, symptoms of HAIs may occur at the time of patient's hospitalization or after discharge (4). According to the World Health Organization (WHO) report, hundreds of millions of people are affected by HAIs every year throughout the world (5). Studies showed that the HAIs rates vary worldwide. In high-income countries, HAIs prevalence in hospitalized patients was $7.6 \%$. This figure was $10.1 \%$ (varied from $5.7 \%$ to $19.1 \%$ ) in low-and middle-income countries (6). Annually, roughly 2 and 4.5 million HAIs are reported in the United States (US) and the European Union, respectively (7). The infections result in 100,000 deaths and impose additional medical care costs of about $\$ 6.5$ billion annually in the US $(6,7)$. According to the WHO's report on 2001, hospital infection has the highest percentage in South-East Asia and the Eastern Mediterranean. Based on this report, one of the main reasons for HAIs is inadvertent misuse of antibiotics leading to widespread resistance. Unfortunately, hospitals in developing countries are hotbeds of infection transmission. These infections lead to increased mortality, longer periods of hospitalization cause emotional and mental stress, failure of surgeries, rejection of organ transplantation and a significant financial burden for healthcare systems and patients. Moreover, they are linked to the spread of multi-drug resistance (MDR) in pathogenic bacteria $(8,9)$.

The most important bacteria causing HAIs are Escherichia coli (E. coli), Klebsiella, methicillin-resistant Staphylococcus aureus (MRSA), Pseudomonas aeruginosa, and Enterococci $(9,10)$. The most common nosocomial infections are urinary tract infections, surgical wound infections, pneumonia, and septicemia (11). Risk factors of the infections for hospitalized patients are divided into two categories: unavoidable risk factors (including old age and serious underlying causes of one's hospitalization) and risk factors that can be mitigated by appropriate treatment (including a longer period of hospitalization, use of inappropriate catheters, excessive use of broad-spectrum, prolonged use of fixed catheters, and improper hand hygiene by healthcare workers) (12).

Prevention of hospital infections is a key way to improve the quality of healthcare. Detailed information on the extent of these infections is essential for evaluating current infection prevention activities and planning for further intervention in the hospitals nationally. An overall review of the documents shows that the reported incidence of all types of HAIs in Iran is very different; so a systematic review of all the documents and their combinations can provide a complete picture of the dimensions of this problem in Iranian society, as well as increase the use of the best and the highest quality documents available. In fact, the purpose of this study was to determine a comprehensive estimate of the prevalence of HAIs, affective factors, and types of these infections in Iran. Our study updates a systematic review that was published in 2018 (13).

\section{Methods}

A systematic and meta-analysis study was done in 2020 . Relevant studies were found in PUBMED, EMBASE, SCOPUS and WEB OF SCIENCES as international databases and Magiran, SID and Medlib as Persian databases from 1995 to September 2020. The following search terms were used: ('Nosocomial infection [Mesh] OR "' hospital infection [Mesh] OR Hospital acquired infection [Mesh] OR health care associated infection 'AND ('Iran' [Mesh]). Also, the references of identifies papers were studied and if their title were in line with the topic, they were investigated by the authors.

The searches were done from July to September 2020. The observational studies (cohorts, case-control, and cross-sectional) both in English and Persian Language were investigated.

Inclusion criteria were: 1) population-based observational studies reporting the prevalence of HAIs, 2) crosssectional, retrospective and case-control studies, and 3) relevant studies with clear and detailed data. Also, case reports, case series, editorials, letters to the editor, commentaries, reviews and clinical trials as well as studies that were not calculating the prevalence of HAIs, were excluded.

Abstract of all papers were imported into Endnote software version 16 then the duplicates were removed. After that, the authors read the full text, and if they had the inclusion criteria, they were kept for more investigation.

Also, the review and editorial articles were excluded. The checklist was prepared by examining the content of the Preferred Reporting Items for Systematic Reviews and Meta-Analyses (PRISMA)(14). The PRISMA Statement comprises a 27-item checklist and a four-phase flow diagram. The checklist includes items considered essential for the transparent reporting of a systematic review. In this Explanation and Elaboration document, the meaning and rationale for each checklist item were explained. For each item, an example of good reporting was included and, anywhere possible, references to pertinent empirical studies and methodological literature.

In the next stage, we checked the results of the papers. If they had reported the rate of nosocomial infection, they were kept as the final suitable papers for analysis.

\section{Data extraction}

A data sheet was created in the Excel software and imported the data of suitable variables. The extracted data were as follows: title, year of the study, HAIs rate, gender of patients, type of infection and bacteria, setting, type of hospital, and sample size.

The search generated a total of 1320 records, of which 602 papers were duplicated, and 718 titles and abstracts 


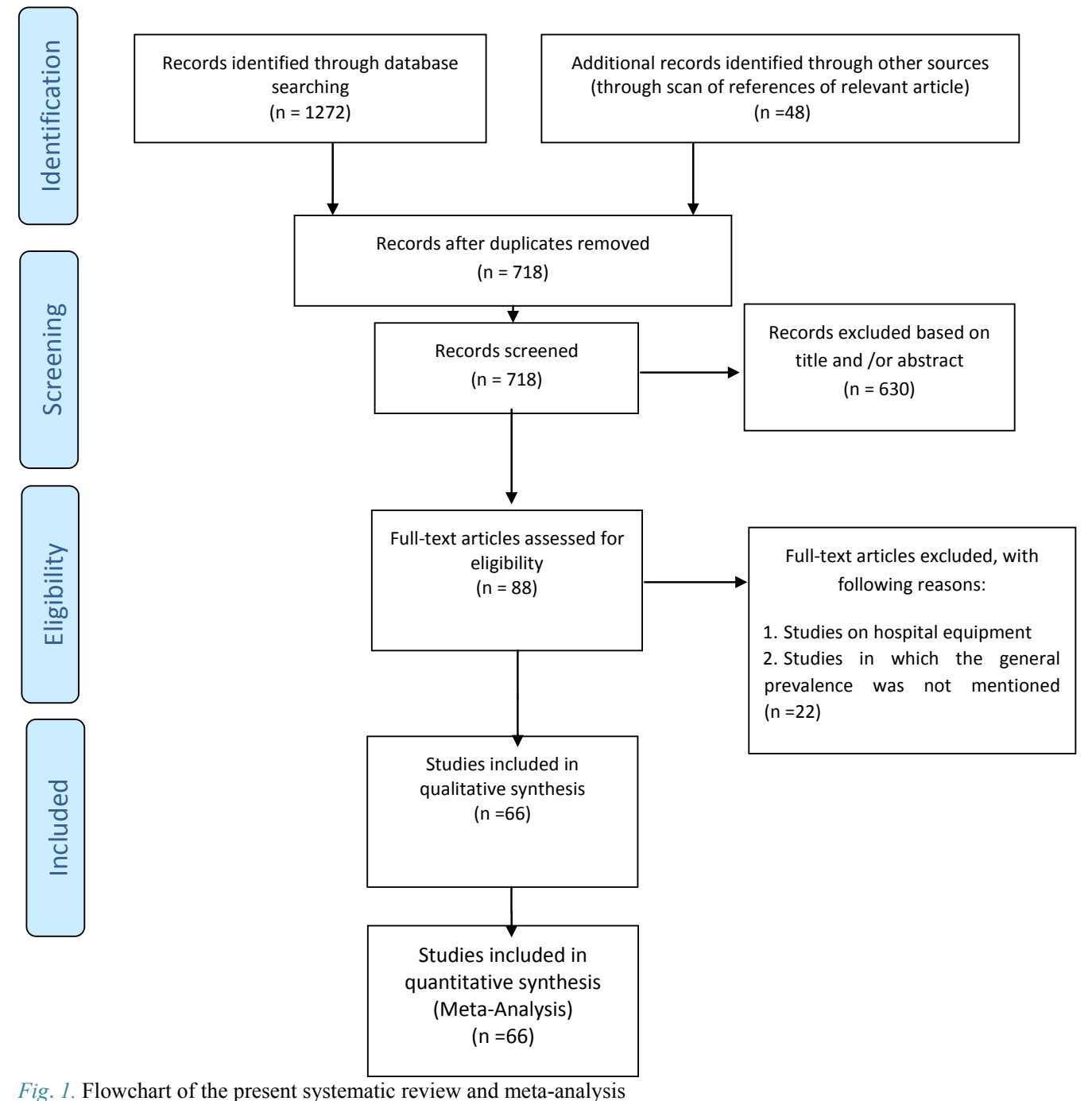

Fig. 1. Flowchart of the present systematic review and meta-analysis

were reviewed. The most fundamental reasons for omission were: studies conducted outside Iran, publication type, and studies not reporting HAIs' rate. A total of 66 articles were included in the meta-analysis and data were extracted. Supplementary information can be accessed in the Preferred Reporting Items for Systematic Reviews and Meta-Analyses 2009 flow diagram (Fig. 1). Also, for assessing the risk of bias, we used ROBVIS as a web app designed for visualizing risk-of-bias.

\section{Data analysis}

Data imported into the STATA software version 11 for analysis. To identify the pooled prevalence, the stochastic DerSimonian-Laird model was applied, computing the effect size with its $95 \%$ confidence interval (CI) and pictorially representing it through a Forest plot. Combined estimates were obtained for the HAIs' overall and in detail by means of random effect models. RR Pooled data were used for the data analysis and mean instruction was used in STATA software. To further examine the source of heterogeneity, meta-regression analyses stratified by publication year and sample size in the hospital. Subgroup analyses were conducted based on study quality, geo- graphic areas, sample size, year of publication, type of infection and hospital. Additionally, the possible sources of heterogeneity were examined using $\mathrm{I}^{2}$ statistics and Cochran's Q test (15). Also, Begg's Rank correlation test and Egger's regression method were used to measure the propagation bias.

\section{Results}

\section{Quality assessment of studies}

The quality assessment of the studies was appraised by the PRISMA checklist. As shown in Figure 1. 66 studies entered to meta-analysis phase. The studies are shown in Table 1.

As shown in Table 2, the odds ratio of hospital infections was reported 0.111 in the studies hospitals in Iran. Although Ecoli was investigated in 53 papers, it found 0.239 in the hospitals. Respiratory infections were reported 0.296 in the studies. In addition, more of the nosocomial infection was described in the South and West of Iran (0.231 and 0.164 , respectively). As a result, male patients had acquired infection more than female (about 0.239 vs. 0.216).

Pooled estimate of HAIs in Iran is shown in Figure 2. 


\begin{tabular}{|c|c|c|c|c|c|c|c|c|}
\hline Author & Year & Setting & $\begin{array}{l}\text { Type of } \\
\text { hospital }\end{array}$ & $\begin{array}{c}\text { Sample } \\
\text { size }\end{array}$ & $\begin{array}{c}\text { HAIs } \\
\text { rate }\end{array}$ & $\begin{array}{c}\text { HAIs in } \\
\text { men }\end{array}$ & $\begin{array}{l}\text { HAIs in } \\
\text { females }\end{array}$ & $\begin{array}{c}\text { Quality } \\
\text { Study }\end{array}$ \\
\hline Rastegegar lari et al.(16) & 1998 & Tehran & Public & 6329 & 0.532 & N/A & N/A & Low \\
\hline Talebi Taher et al.(17) & 2001 & Qazvin & Public & 546 & 0.041 & $\mathrm{~N} / \mathrm{A}$ & N/A & Low \\
\hline Shojaee et al.(18) & 2002 & Shahrekord & Public & 845 & 0.049 & N/A & N/A & Low \\
\hline Samadzadeh et al.(19) & 2002 & Oromea & Public & 942 & 0.049 & N/A & N/A & Low \\
\hline Askarian et al.(20) & 2003 & Shiraz & Public & 106 & 0.451 & N/A & N/A & Medium \\
\hline Ekrami et al.(21) & 2005 & Ahwaz & Public & 182 & 0.769 & N/A & N/A & Medium \\
\hline Sadegh Zadeh et al.(22) & 2005 & Zanjan & Public & 150 & 0.025 & N/A & N/A & High \\
\hline Mousavian et al.(23) & 2006 & Ahwaz & Public & 1604 & 0.044 & N/A & N/A & High \\
\hline Qurbanalizadegan et al.(24) & 2006 & Tehran & Public & 6817 & 0.013 & N/A & N/A & Medium \\
\hline Gorbanalizadegan et al.(25) & 2006 & Tehran & Public & 155 & 0.039 & N/A & N/A & Medium \\
\hline Naderi Nasab et al.(26) & 2006 & Mashhad & Public & 1341 & 0.039 & N/A & N/A & Medium \\
\hline Nik Bakht et al (27) & 2007 & Tabriz & Public & 460 & 0.348 & 0.333 & 0.358 & Low \\
\hline Ajal Loeyan et al.(28) & 2007 & Tehran & Private & 234 & 0.183 & N/A & N/A & Medium \\
\hline Esmaili et al.(29) & 2007 & Tehran & Public & 116 & 0.017 & N/A & N/A & Medium \\
\hline Mohmmadi Mehr et al.(30) & 2008 & Tehran & Public & 165 & 0.393 & N/A & N/A & Medium \\
\hline Sharifi et al.(31) & 2008 & Qazvin & Public & 1083 & 0.052 & N/A & N/A & Medium \\
\hline Ghazvini et al.(32) & 2008 & Mashhad & Public & 971 & 0.033 & N/A & N/A & Medium \\
\hline Oskouee et al.(33) & 2009 & Tabriz & Public & 103 & 0.331 & N/A & N/A & Medium \\
\hline Asgare Moghadam et al.(34) & 2009 & Tehran & Public & 181 & 0.741 & N/A & N/A & Medium \\
\hline Amini et al.(35) & 2009 & Tehran & Private & 691 & 0.109 & N/A & N/A & Medium \\
\hline Talaie et al.(36) & 2010 & Tehran & Public & 582 & 0.08 & N/A & N/A & Medium \\
\hline Darvishpor et al.(37) & 2010 & Rasht & Public & 270 & 0.163 & $\mathrm{~N} / \mathrm{A}$ & N/A & High \\
\hline Khani et al.(38) & 2011 & Tehran & Public & 256 & 0.341 & 0.323 & 0.359 & Medium \\
\hline Afkhamzadeh et al.(39) & 2011 & Sanandaj & Public & 149 & 0.322 & 0.237 & 0.351 & Medium \\
\hline Larypoor et al.(40) & 2011 & Qom & Public & 29631 & 0.001 & N/A & N/A & Low \\
\hline Barak et al.(41) & 2011 & Tehran & Public & 1795 & 0.039 & N/A & N/A & Medium \\
\hline Ghorbani Birgani et al.(42) & 2011 & Ahwaz & Public & 772 & 0.101 & 0.129 & 0.075 & Medium \\
\hline Mobin et al.(43) & 2012 & Hamedan & Public & 353 & 0.171 & N/A & N/A & High \\
\hline Saedi et al.(44) & 2012 & Mashhad & Public & 647 & 0.172 & N/A & N/A & Medium \\
\hline Pourakbari et al.(45) & 2012 & Tehran & Public & 1497 & 0.034 & N/A & N/A & Low \\
\hline Ghazvini et al.(32) & 2012 & Mashhad & Public & 971 & 0.033 & N/A & N/A & Low \\
\hline Soltani et al.(46) & 2012 & Tehran & Public & 464 & 0.373 & N/A & N/A & Medium \\
\hline Hashemi et al.(47) & 2013 & Hamedan & Public & 574 & 0.528 & N/A & N/A & Low \\
\hline $\begin{array}{l}\text { Heydari Sour Shojaee et } \\
\text { al.(48) }\end{array}$ & 2013 & Charmahale bakhtyare & Public & 848 & 0.087 & N/A & N/A & Medium \\
\hline Shojaei et al.(49) & 2013 & Qom & Public & 12668 & 0.076 & N/A & N/A & Low \\
\hline Saadat et al.(50) & 2013 & Shiraz & Public & 591 & 0.149 & N/A & N/A & Medium \\
\hline Abedini et al.(51) & 2014 & Kurdistan & Public & 369 & 0.027 & N/A & N/A & Medium \\
\hline Akhavan Tafti et al.(52) & 2014 & Yazd & Public & 180 & 0.003 & N/A & N/A & Medium \\
\hline Shakib et al.(53) & 2014 & Sanandaj & Public & 750 & 0.103 & $\mathrm{~N} / \mathrm{A}$ & N/A & Low \\
\hline Davodi et al.(54) & 2014 & Mazandaran & Public & 5712 & 0.010 & N/A & N/A & Low \\
\hline Makhloghi et al.(55) & 2014 & Qazvin & Public & 188 & 0.196 & N/A & N/A & Low \\
\hline Bijari et al.(56) & 2014 & South Khorasan & Public & 39777 & 0.001 & N/A & N/A & Low \\
\hline Behzadnia et al.(57) & 2014 & Mazandaran & Public & 34556 & 0.102 & N/A & N/A & Medium \\
\hline Saeidimehr et al.(58) & 2015 & Ahwaz & Private & 16936 & 0.020 & N/A & N/A & Medium \\
\hline Hashemizadeh et al.(59) & 2015 & Shiraz & Public & 2229 & 0.114 & $\mathrm{~N} / \mathrm{A}$ & $\mathrm{N} / \mathrm{A}$ & Medium \\
\hline Haje bageri et al.(60) & 2015 & Sanandaj & Public & 160 & 0.152 & 0.174 & 0.132 & High \\
\hline Hosini et al.(61) & 2016 & Jahrom & Public & 189 & 0.254 & N/A & N/A & High \\
\hline Servatyare et al.(62) & 2017 & Sanandaj & Public & 198 & 0.167 & 0.162 & 0.172 & Medium \\
\hline Rahmanian et al.(63) & 2017 & Jahrom & Public & 55295 & 0.002 & N/A & N/A & High \\
\hline Shali et al.(64) & 2017 & Tehran & Public & 300 & 0.035 & N/A & N/A & High \\
\hline Farzanpour et al.(65) & 2017 & Sabzevar & Public & 89429 & 0.012 & N/A & N/A & Medium \\
\hline Dadmanesh et al.(66) & 2017 & Tehran & Public & 900 & 0.472 & N/A & N/A & High \\
\hline Heydarpour et al.(67) & 2017 & Kermanshah & Public & 6000 & 0.023 & N/A & N/A & High \\
\hline Eshrati et al.(68) & 2018 & Iran & Public & 7018393 & 0.012 & N/A & N/A & High \\
\hline Ghanbari et al.(69) & 2018 & Isfahan & Public & 5500 & 0.045 & N/A & N/A & Medium \\
\hline Nasiri et al.(70) & 2018 & Tehran & Public & 11164 & 0.033 & N/A & N/A & Medium \\
\hline Rahimi-Bashar et al.(71) & 2018 & Hamedan & Public & 10332 & 0.026 & N/A & N/A & Medium \\
\hline Kohestani et al.(72) & 2019 & Tehran & Public & 600003 & 0.046 & N/A & N/A & Low \\
\hline Alkhudhairy et al.(73) & 2019 & Ahvaz & Public & 380 & 0.316 & N/A & N/A & High \\
\hline Azimi et al.(74) & 2019 & Tehran & Public & 14690 & 0.077 & N/A & N/A & Medium \\
\hline Piruozi et al.(75) & 2019 & Grash & Public & 300 & 0.068 & $\mathrm{~N} / \mathrm{A}$ & $\mathrm{N} / \mathrm{A}$ & Medium \\
\hline Yaqubi et al.(76) & 2019 & Rasht & Public & 738 & 0.057 & N/A & N/A & High \\
\hline Sepandi et al.(77) & 2019 & Tehran & Public & 14517 & 0.017 & N/A & N/A & High \\
\hline Mansori et al.(78) & 2020 & Mashhad & Public & 2800 & 0.411 & N/A & N/A & High \\
\hline Ahmadinejad et al.(79) & 2020 & Kerman & Public & 197 & 0.401 & N/A & N/A & High \\
\hline Emami et al.(80) & 2020 & Shiraz & Public & 3420 & 0.281 & N/A & N/A & High \\
\hline
\end{tabular}

According to Table 3. HAIs in the south of Iran are analysis. vaired between 0.002 to 0.451 .

Egger's test was done to evaluate publication bias (Fig. Table 4 shows the results of the meta-regression 
Table 2. The results of sub-groups analysis of hospital infection in Iranian hospitals from 1995-2020

\begin{tabular}{|c|c|c|c|c|}
\hline Variable & No. reports & Pooled $(95 \% \mathrm{CI})$ & $\mathrm{I} 2(\%)$ & $\mathrm{p}$ \\
\hline \multicolumn{5}{|l|}{ Quality of studies } \\
\hline High & 16 & $0.193(0.143-0.242)$ & $99.9 \%$ & 0.001 \\
\hline Medium & 35 & $0.097(0.089-0.106)$ & & \\
\hline Low & 15 & $0.120(0.104-0.137)$ & & \\
\hline \multicolumn{5}{|l|}{ Time } \\
\hline$<2014$ & 43 & $0.157(0.142-0.172)$ & $99.9 \%$ & 0.001 \\
\hline$>2014$ & 23 & $0.089(0.082-0.097)$ & & \\
\hline \multicolumn{5}{|l|}{ Patients' gender } \\
\hline Male & 6 & $0.239(0.160-0.318)$ & $99.7 \%$ & $>0.5$ \\
\hline Female & 6 & $0.216(0.124-0.307)$ & & \\
\hline \multicolumn{5}{|l|}{ Age } \\
\hline$\leq 50$ & 44 & $0.117(0.111-0.124)$ & $99.6 \%$ & $>0.5$ \\
\hline$>50$ & 22 & $0.111(0.098-0.123)$ & & \\
\hline \multicolumn{5}{|l|}{ Sample Size } \\
\hline$\leq 1500$ & 42 & $0.191(0.162-221)$ & $99.9 \%$ & 0.001 \\
\hline$>1500$ & 24 & $0.087(0.080-0.095$ & & \\
\hline \multicolumn{5}{|l|}{ Region } \\
\hline Center & 30 & $0.124(0.112-0.136)$ & $99.5 \%$ & 0.001 \\
\hline East & 7 & $0.083(0.071-0.096)$ & & \\
\hline North & 4 & $0.081(0.017-0.142)$ & & \\
\hline South & 7 & $0.231(0.121-0.341)$ & & \\
\hline West & 19 & $0.164(0.143-0.185)$ & & \\
\hline \multicolumn{5}{|l|}{ Type of Hospital } \\
\hline Public & 63 & $0.112(0.107-0.118)$ & $97.9 \%$ & $>0.5$ \\
\hline Private & 3 & $0.100(0.015-0.186)$ & & \\
\hline \multicolumn{5}{|l|}{ Type of bacteria } \\
\hline Staphylococcus aureus & 49 & $0.166(0.151-0.182)$ & $99.9 \%$ & 0.001 \\
\hline klebsiella & 52 & $0.180(0.163-0.196)$ & & \\
\hline Escherichia coli & 53 & $0.239(0.212-0.266)$ & & \\
\hline \multicolumn{5}{|l|}{ Type of infection } \\
\hline Respiratory & 38 & $0.286(0.261-0.310)$ & $99.9 \%$ & $>0.5$ \\
\hline Urinary & 51 & $0.296(0.255-0.337)$ & & \\
\hline Overall & 66 & $0.111(0.105-0.116)$ & $99.9 \%$ & \\
\hline
\end{tabular}

$(\mathrm{p}=0.001)$.

\section{Discussion}

HAIs have always been a major health problem as hospitals expand that, despite multiple attempts, no country or organization has managed to fully resolve (81). In this systematic review and meta-analysis, we have shown that the overall prevalence of HAIs in Iran was 0.111 (95\% CI: $0.0 .105-0.116)$. The previously systematic review by Ghashghaee et al. (13). revealed the HAIs rate in Iran was $4.5 \%$. HAIs rates are also $10.1 \%$ (varied from $5.7 \%$ to $19.1 \%$ ) in developing countries and $7.6 \%$ (varied from $3.5 \%$ to $12 \%$ ) in developed countries (5). According to WHO reports, the HAIs rate is between $5 \%-22 \%$ in the world (82). The high prevalence of HAIs in Iran highlights the need for urgent attention and implementation of a comprehensive plan to control these infections. Variations in HAI rates can, however, be due to differences in diagnostic criteria and tests for infection diagnosis, as well as differences in reporting systems and their consistency.

According to the findings, the HAIs had decreased from 0.157 to 0.089 after HTP in Iran, which was statistically significant $(p=0.001)$. This indicates that the measures taken in HTP have affected the quality of health services and reduced nosocomial infections. In this regard, Ghashghaee in his study, found that HAIs had decreased from $7.6 \%$ to $2.4 \%$ after HTP (13). Moreover, Braithwaite et al. in their Book in 2018 (83) reported that In Iran, a government policy initiative called HTP was implemented to decrease inequality and improve public health coverage and reached more objectives especially patient safety.

Based on our measurement, the most common bacteria causing HAIs were Escherichia coli and Klebsiella. The findings also demonstrated that respiratory infections and urinary infections were the most common HAIs. These findings are supported by studies carried out in EMRO (the Eastern Mediterranean Regional Office of the World Health Organization) (82). Moreover, a meta-analysis study in Iran reported Klebsiella as common bacteria in HAIs (13). These infections are directly related to contamination of equipment, especially urinary catheters, environment and operating room personnel, and air conditioning systems, which in many developing countries is due to lack of proper equipment.

Our findings showed that the male is more likely to have HAIs than the female. A similar result was reported in the systematic review study conducted in EMRO (82). Clinicians should be mindful of these differences and take them under consideration when managing patients with HAIs. However, one of the probable reasons could also be the lower number of women surveyed in the total papers reviewed in the present study.

According to the results, the prevalence of HAIs in the south and west was more than in other regions of the country. The high rate of HAIs in some parts of the country is the characteristics of studied patients, their underlying diseases and the hospitalized ward. Most of the patients in south Iran were hospitalized in intensive care 


\begin{tabular}{|c|c|c|c|}
\hline $\begin{array}{l}\text { Study } \\
\text { is }\end{array}$ & & Es Coss CD & $\begin{array}{l}\text { \& } \\
\text { Woight }\end{array}$ \\
\hline otranti et el. [2005] & 1 & $0.77(0.71,0.83)$ & 0.55 \\
\hline Aspoes magedem at el. [2000) & i & $0.74(0.68,0.80)$ & 0.52 \\
\hline Raneger Lei as al (1005) & 1 & $0.53(0.52,0.54)$ & 190 \\
\hline Heahemi an al (2013) & i & $0.53(0.42,0.57)$ & 0.93 \\
\hline Dodmenesh an al (2017) & i & $0.47(0.46,0.50)$ & 1.17 \\
\hline Esekrien at el. (20035) & i & 0.45 (10.36,0.55) & 0.27 \\
\hline Maraeri at at (2020) & i & $0.41(0.30,0.43]$ & 1.60 \\
\hline Ahmedinged of at: (2020) & i & $0.40(033,0.9)$ & 0.46 \\
\hline Mehrredimaty at al (2008) & i & $0.30(0.32,0.97)$ & 0.40 \\
\hline Sehar iot ed. (20012) & i & $0.37(0.33,0.42)$ & 0.25 \\
\hline Natei et al (2007) & i & $0.35(0.30,0.39)$ & 0.87 \\
\hline Wheni at al (2011) & i & $0.34(0.28,0.49)$ & 0.50 \\
\hline Oabouses at at (2000) & i & $0.33(0.24,0.42)$ & 0.20 \\
\hline Afechemrabeh on al (2011) & i & $0.32025,0.40$ & 0.40 \\
\hline 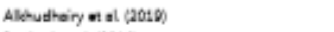 & 1 & $0.32(0.27,0.36$ & 0.70 \\
\hline Bophes ind al (2014) & i & $0.30(0.30,0.391$ & 200 \\
\hline Emani ot at (20080) & i & $0.28(0.27,0.30)$ & 1.90 \\
\hline Hesini at at (2016) & i & $0.25(0.10,0.32)$ & 0.54 \\
\hline Maethioghi at el. [2014] & & $0.20(0.14,0.25)$ & 0.61 \\
\hline Abea layen at el (20007) & 1 & $0.18(013,0.23)$ & 0.74 \\
\hline Sees at el. (2012)] & $i$ & $0.170 .14,0.20$ & 1.20 \\
\hline Matis at d. (2012) & & $0.17(013,0.29)$ & 0.97 \\
\hline Servetyoues at al (2017) & 0 & $0.17(0.12,0.22)$ & 0.60 \\
\hline Derrinherer at al: (2010) & - & $0.16(0.12,0.29$ & 0.85 \\
\hline Hoje tegen at ed (2015) & 1 & $0.16(0.10,0.29)$ & 0.62 \\
\hline So'edet an al (2013) & & $0.15(0.12,0.17)$ & 1.31 \\
\hline Teleic et el. [2010] & 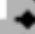 & $0.12(0.10,0.15]$ & 1.37 \\
\hline Heatemitosteh of al. (2015) & $\bullet$ & $0.11(0.10,0.13)$ & 1.87 \\
\hline Amini at d. (2000) & $\bullet$ & $0.11(0,00,0.13)$ & 150 \\
\hline Shatibe et al (20:4) & $\bullet$ & 0.10 (pos, 0.12 & 1.55 \\
\hline Behasodnis ot el (2014) & $\bullet$ & $0.10(0.10,0.19)$ & 2.10 \\
\hline Ghorbari at el. (2011) & $\bullet$ & $0.20 \cos 0.0 .128$ & 157 \\
\hline Hoydari Sour S-ajame ne al (2013) & 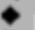 & $0.00(0.07,0.19$ & 1.66 \\
\hline Astmi nat at (2018) & $\bullet$ & $0.08(0.07,0.00)$ & 200 \\
\hline Shojesti is al: (2013) & $\bullet$ & $0.06(0.07,0.005)$ & 206 \\
\hline Direst in al (2010) & 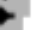 & $0.07 \cos 0.0 .10$ & 1.31 \\
\hline Yoguti es al: (2010) & & $0.06(000,0.07)$ & 1.74 \\
\hline Sherifi an al (2006) & 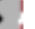 & $0.05(0.040 .07)$ & 1.87 \\
\hline Shojeen et el. (2002) & 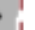 & $0.05(0.03,0.06$ & 1.82 \\
\hline Semedesdehet d. (2002) & 1 & $0.05(0.03,0.06)$ & 1.85 \\
\hline Keet int di. (2019) & 1 & $0.05(0.05,0.05)$ & 211 \\
\hline Botsodnis ot at (2018) & 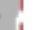 & $0.05(\cos , 0.05)$ & 2007 \\
\hline Mountrian at al (2006) & 1 & $0.04(0.03,0.05)$ & 1.96 \\
\hline Telotei Tether as al (200) & 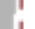 & $0.04(0.02,0.06)$ & 1.75 \\
\hline Nasteri Nereb of al: (2015) & 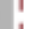 & $.004(0.03,0.05]$ & 1.95 \\
\hline Ghorbanaitedegen at at. (2006) & 1 & O.0. (p.01,0.07) & 1.24 \\
\hline Bartek en al (2011) & ! & $0.04(0.03,0.05)$ & 1.90 \\
\hline Shat at ed. (2017) & 1 & $0.0400 .01,0.06\}$ & 1.50 \\
\hline Dourtitteri is al (2012) & I & $0.08000,0.04$ & 190 \\
\hline Ghasvisi at al (2008) & 1 & $0.080002,0.04$ & 198 \\
\hline Gesvin et ad. (2012) & 1 & $0.0500 .02,0.04$ & 1.98 \\
\hline Naniri et el (2018) & 1 & $0.05(0,03,0.06)$ & 2.10 \\
\hline Abedini an al (2014) & 1 & $0.080001,0.04$ & 175 \\
\hline Ratimi-Banter on al (2018) & 1 & $0.08(0.02,0.03)$ & 2.10 \\
\hline Sedogh Zosteh on al (2005) & 1 & $0.05(0,00,0.05)$ & 1.43 \\
\hline Heydarpour on at (2017) & I & $0.02(0.02,0.03)$ & 200 \\
\hline Seedi-etr an al (2015) & 1 & $0.02(0.02,0.02)$ & $2: 11$ \\
\hline àmeñ et al (2007) & I & $0.02(-1001,000)$ & 1.40 \\
\hline Sesondi in al (2010) & 1 & 0.02 (pont, 0.028 & $2: 11$ \\
\hline Ghorbanaitedegen at at. (2006) & 1 & $0.01(0.01,0.02)$ & 2.10 \\
\hline Ferrengeur of at (2017) & 1 & $0.01001,0.09$ & 211 \\
\hline tatrati and (2018) & 1 & $0.01001,0.09$ & $2: 11$ \\
\hline Denedi at at (2014) & 1 & $001001,0.09$ & 210 \\
\hline loriesert at el. (2011) & 1 & $0.000000,0.00$ & 211 \\
\hline Akthenen Tots at di. (2014) & 1 & $0,000(-1,00,004)$ & 202 \\
\hline Rahterien at al (2017) & 1 & $0,000000,0.00)$ & 211 \\
\hline Bjerti at at (2014) & 1 & $0.000000,0.000$ & 211 \\
\hline 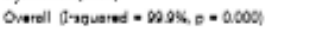 & 1 & $0.11(0.11,0.12)$ & 100.00 \\
\hline 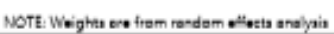 & & & \\
\hline
\end{tabular}

Fig. 2. The forest plot of the overall prevalence of nosocomial infections in Iran

units $(20,79)$. Moreover, Ahmadinejad declared that about $50 \%$ of the studied patients were addicts and they are prone to nosocomial infections (79).

Monitoring and controlling HAIs is difficult, costly, and time-consuming; however, it is necessary and costeffective. Adherence to hygiene principles and methods of microbiological diagnosis can prevent and control HAIs with lower costs. Using minimally invasive devices and methods, paying close attention to non-intravenous nutrition, preventing misuse and overuse of antibiotics, moni- toring the pattern of infection, improving hospital environmental health, training personnel, and effective hand hygiene strategies are methods that can significantly reduce HAIs $(84,85)$.

\section{Conclusion}

According to the reviewed studies, the prevalence of HAIs in Iran is relatively high. Despite the increasing development of health standards and quality development of hospitals in recent years, the prevalence findings indicate 
Table 3. The overall prevalence of nosocomial infections in the south of Iran

\begin{tabular}{|c|c|c|c|}
\hline Study & 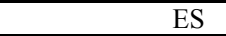 & $95 \%$ Conf. Interval & Weight \\
\hline Askarian et al. & 0.451 & $0.356-0.546$ & 13.28 \\
\hline Ahmadinejad et al. & 0.401 & $0.333-0.469$ & 13.94 \\
\hline Emami et al. & 0.281 & $0.266-0.296$ & 14.69 \\
\hline Hosini et al. & 0.254 & $0.192-0.316$ & 14.07 \\
\hline Saadat et al. & 0.146 & $0.118-0.174$ & 14.59 \\
\hline Hashemizadeh et al. & 0.114 & $0.101-0.127$ & 14.70 \\
\hline Rahmanian et al. & 0.002 & $0.002-0.002$ & 14.72 \\
\hline Pooled ES & 0.231 & $0.121-0.341$ & 100.00 \\
\hline \multicolumn{4}{|c|}{ Table 4. Results of the meta-regression } \\
\hline Overall prevalence & Coef. & Std. Error & $\mathrm{p}$ \\
\hline Year of publication & -0.006 & -1.49 & 0.141 \\
\hline Region & 0.008 & 0.018 & 0.670 \\
\hline Type of hospital & -0.070 & -0.65 & 0.516 \\
\hline
\end{tabular}

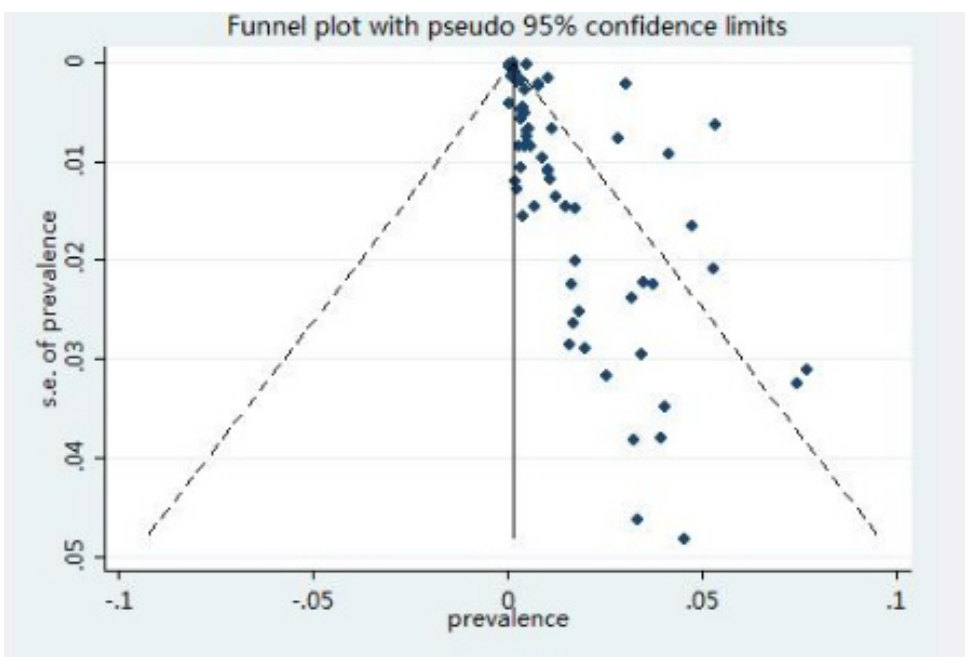

Fig. 3. The Egger test for publication bias

Table 5. The Egger test for publication bias

\begin{tabular}{|c|c|c|c|c|c|}
\hline Std Eff & beta coefficient. & Std. Err & $\mathrm{t}$ & $\mathrm{p}$ & $95 \%$ Conf. Interval \\
\hline Slope & .0107469 & .001112 & 9.66 & 0.001 & .0085242 .0129696 \\
\hline bias & 13.41402 & 3.581168 & 3.75 & 0.001 & $6.261939 \quad 20.56611$ \\
\hline
\end{tabular}

a relatively uneven trend in this development and an increase in the prevalence of HAIs in different parts of the country. This should be taken into consideration by health policymakers for pathology and revision of some previous programs and standards as well as the development of appropriate and evidence-based control and education programs to reduce this health problem in Iran. Efforts to improve the quality of nursing care, applied staff training, continuous monitoring HAIs, provision of facilities, implementation of infection control programs, prioritization of hospital wards for more stringent health measures, emphasis on preventive cares such as hand washing and appropriate training through mass media are the most important actions suggested in this regard.

\section{Acknowledgment}

Thanks to Zahedan University of Medical Sciences.

\section{Conflict of Interests}

The authors declare that they have no competing interests.

\section{References}

1. Medici A, Murray R. Hospital performance and health quality improvements in São Paulo (Brazil) and Maryland (USA). 2010.

2. Klompas M. Epidemiology, pathogenesis, microbiology, and diagnosis of hospital-acquired and ventilator-associated pneumonia in adults. UpToDate Waltham, MA: UpToDate Inc http://www uptodate com (Accessed on August 11, 2018). 2018.

3. Ahoyo TA, Bankolé HS, Adéoti FM, Gbohoun AA, Assavèdo S, Amoussou-Guénou M, et al. Prevalence of nosocomial infections and anti-infective therapy in Benin: results of the first nationwide survey in 2012. Antimicrob Resist Infect Control. 2014;3(1):17.

4. Luzzatto L, Longo D, Fauci A, Kasper D, Hauser S, Jameson J, et al. Harrison's principles of internal medicine, 19e. 2015. Mcgraw-hill Publisher.

5. Kilpatrick C, Allegranzi B, Pittet D. WHO First Global Patient Safety Challenge: Clean Care is Safer Care. Contributing to the training of health-care workers around the globe. Int $\mathrm{J}$ Infect Control. 2011;7(2):17.

6. World Health Organization. WHO Guidelines on Hand Hygiene in Health Care: a Summary First Global Patient Safety Challenge Clean Care Is Safer Care. 2009.

7. Gesser-Edelsburg A, Cohen R, Halavi AM, Zemach M, van Heerden PV, Sviri S, et al. Beyond the hospital infection control guidelines: a qualitative study using positive deviance to characterize gray areas and to achieve efficacy and clarity in the prevention of healthcareassociated infections. Antimicrob Resist Infect Control. 2018;7(1):1- 
10.

8. Drohan SE, Levin SA, Grenfell BT, Laxminarayan R. Incentivizing hospital infection control. Proceed Natl Acad Sci U S A. 2019;116(13):6221-5.

9. Organization WH. Prevention of hospital-acquired infections: a practical guide. Geneva, Switzerland: World Health Organization, 2002.

10. Khan HA, Baig FK, Mehboob R. Nosocomial infections: Epidemiology, prevention, control and surveillance. Asian Pac J Trop Biomed 2017;7(5):478-82.

11. Allegranzi B, Nejad SB, Combescure C, Graafmans W, Attar H, Donaldson L, et al. Burden of endemic health-care-associated infection in developing countries: systematic review and metaanalysis. Lancet. 2011;377(9761):228-41.

12. Cecil RLF, Goldman L, Schafer AI. Goldman's Cecil Medicine, Expert Consult Premium Edition--Enhanced Online Features and Print, Single Volume, 24: Goldman's Cecil Medicine: Elsevier Health Sciences; 2012

13. Ghashghaee A, Behzadifar M, Azari S, Farhadi Z, Bragazzi NL, Behzadifar M, et al. Prevalence of nosocomial infections in Iran: A systematic review and meta-analysis. Med J Islam Repub Iran. 2018;32:48.

14. Liberati A, Altman DG, Tetzlaff J, Mulrow C, Gøtzsche PC, Ioannidis JP, et al. The PRISMA statement for reporting systematic reviews and meta-analyses of studies that evaluate health care interventions: explanation and elaboration. $\mathrm{J}$ Clin Epidemiol 2009;62(10):e1-e34.

15. Aryankhesal A, Behzadifar M, Bragazzi NL, Ghashghaee A, Behzadifar M. A framework for conducting meta-analysis studies; methodological concerns and recommendations. Iran J Public Health. 2018;47(5):773-4.

16. Lari AR, Honar HB, Alaghehbandan R. Pseudomonas infections in Tohid burn center, Iran. Burns. 1998;24(7):637-41

17. Talebei Taher M, Asef Zade M, Sarreshtedary M. The incidence of nosocomial infections in the internal wards of the nerves, newborns, icu, nicu in educational hospitals affiliated to Qazvin University of Medical Sciences and Health Services. Iran J Infect Dis Trop Med. 2001;6(14):48-52.

18. Shojaee H, Borjian Brojeni S, Arti H, Shirani S. Study of clean (Class I) surgical wound infections in Shahrekord and Borujen hospitals, 2000. J Shahrekord Univ Med Sci. 2002;4

19. Samadzadeh S, Sadeghi A, Sadeghi R, Rahbar M. Determining the frequency and causative factors of nosocomial infections in patients admitted to Imam Khomeini Hospital in Urmia in 2000. J Urmia Univ Med Sci. 2002;13(3):220 - 7.

20. Askarian M, Hosseini SR, Kheirandish P. Incidence and Microorganisms Causing Nosocomial Infections in Ghotbeddin Burn Center of Shiraz, Iran, 2000-2001. J Kerman Univ. Medical Sci. 2003;10(2):65 - 70 .

21. Ekrami A, Kalantar E. Bacterial infections in burn patients at a burn hospital in Iran. Indian Council Med Res. 2007;126(6):541.

22. Sadeghzadeh V, Hassani N. The frequency rate of nosocomial urinary tract infection in intensive care unit patients in Shafiieh Hospital, Zanjan, 2004. J Adv Med Biomed Res. 2005.

23. Moosavian SM, Pordeli HR. Survey of Respiratory and Urogenital Infections Due to Mycoplasma in the Hospitalized Patients in Ahwaz Imam Khomeini Hospital. J Kerman Univ Medical Sci. 2003;10(4):185 - 92.

24. Qurbanalizadgan M, Ranjbar R, Esmaili D. The prevalence of multidrug resistant Staphylococcus aureus in patients admitted to Baqiyatallah Hospital (2005). J Qazvin Univ Medical Sci. 2008;11(4):92-3.

25. Ghorban AM, Ranjbar R, Joneydi JN, Esfahani A, Esmaeili D, Goudarzi Z. A study on the prevalence of nosocomial infections in ICU patients admitted at Baqyiatallah Hospital. J Ilam Univ Med Sci. 2008.

26. Naderi-Nasab M, Farhat A, Tajzadeh P, Sourosh S, Amiri M. Study of the bacterial agents in nosocomial and acquired infections based on the blood culture in neonatal intensive care unit of a hospital, north east of Iran. Saudi Med J. 2007;28(5):723 - 726.

27. Nikbakht M, Nahaei MR, Akhe MT, Asgharzade M, Nikvash S. Frequency of nasopharyngeal carriers with Staphylococcus aureus in medical staff and hospitalized patients and pattern of antibiotic resistance of nasal strains and clinical specimens in Tabriz hospitals. J. Tabriz Univ Medical Sci. 2007;29(2):131-8.
28. Ajan Loeyan M, Kazemi H, Samar G, Feyzade A. The incidence of infection in the intensive care unit and the factors affecting it in Khatam Al-Anbia Hospital in Tehran. J Gorgan Univ Medical Sci. 2007;9(2):24 - 8 .

29. Ghorban AM, Ranjbar R, Izadi M, Esmaeili D, Ahmadi A, Ghoudarzi Z. The Prevalence of multi-Resistant Pseudomonas aeruginosa and Acinetobacter spp. in patients addmited in baqiyatallah hospital in 2005. J Ilam Univ Med Sci. 2007: 15(1): 14-18

30. Mohammadi Mehr M, Feyzabadi MM, Bahadori O, Mohsen Motashkar Arani M, Khosravi M. Evaluation of frequency and determination of antibiotic resistance of gram-negative bacteria responsible for infection Hospital of Intensive Care Unit of Besat Hospital, Tehran. Iran J Microbiol. 2008;3(2):47 - 54.

31. Sharifi M, Karimzadeh T, Mohammadi-Chelkasari F, Bijani B, Alipoor-Heydari M. Community-acquired methicillin-resistant Staphylococcus aureus: prevalence and risk factors. J Inflamm Dis. 2009;12:75-82.

32. Ghazvini K, Rashed T, Boskabadi H, Yazdan Panah M, Khakzadan F, Safaee H, et al. Neonatal intensive care unit nosocomial bacterial infections. Tehran Univ Med J. 2008;66(5):349-54.

33. Oskouie SA, Rezaee MA, Ghabili K, Firoozi F. An epidemiological study of nosocomial infections in tabriz children's hospital based on national nosocomial infection surveillance system (nnis). Life Sci J. 2013;10(1):277-9.

34. Asghari Moghadam N, Talebi M, Hosseini Moghadam SMM, Seifi M, Javadi Gh, Pourshafi MR. Frequency of bacteria causing urinary tract infections and their antibiotic resistance in catheterized patients. Iran J Infect Dis Trop Med. 2009;14(47):33-7.

35. Amini M, Sanjary L, Vasei M, Alavi S. Frequency evaluation of the nosocomial infections and related factors in Mostafa Khomeini Hospital" ICU" based on" NNI" system. Sci Res J Army Univ Med. Sci.. 2009; 7(1):9-14.

36. Talaie H, Sabeti S, Mahdavinejad A, Barari B, Kamalbeik S. A survey on microorganisms and their sensitivity by E-Test in ventilatorassociated pneumonia at Toxicological-Intensive Care Unit of Loghman-Hakim Hospital. Acta Biomed. 2010;81(3):210-6.

37. Darvishpour A, Hashemian H, Faal E, Fasihi M. Survey of nosocomial infection and accompanied factors in neonatal intensive care unit. J Guilan Uni Med Sci. 2010; 19(73): 37-45

38. Khanighaleejogh R, Kaji MA, Shamsi A, Norrighoshki H. Prevalence of Urinary Tract Disorders in Residents of Kahrizak Elderly House. HAYAT. 2011;17(2).

39. Afkhamzade A, Lahorpoor F, Del Pishe A, Janmardi R. The incidence of ventilatorassociated pneumonia and bacterial resistance patterns in the Intensive care adult Besat hospital, Sanandaj, Iran. J Kordestan Univ Med Sci. 2011;16:20-6.

40. Larypoor M, Frsad S. Evaluation of nosocomial infections in one of hospitals of Qom, 2008. Iran J Microbiol. 2011;5(3):7-17.

41. Barak M, Mamishi S, Siadati SA, Salamati P, Khotaii G, Mirzarahimi M. Risk factors and bacterial etiologies of nosocomial infections in NICU and PICU Wards of children's medical center and bahrami hospitals during 2008-2009. J Ardabil Univ Med Sci. 2011;11(2):113-20.

42. Birgani AG, Asadpoor S. Nosocomial infections in intensive care unit of Ahvaz Arya Hospital (2008-2009). Mod Care J. 2011;8(2).

43. Mobaien A, Amirhasani S, Nekoei A, Nekoei B. Study of nosocomial urinary tract infections in the ICUs of Hamadan Besat and Ekbatan Hospitals during the 1387-89 Period. J Adv Med Med Res. 2012;20(79):94-102.

44. Saedi S, Chakerzehi A, Soltani N, Honarmand M, Yazdanpanah M, Ghazvini K, et al. Nosocomial urinary tract infections: etiology, risk factors and antimicrobial pattern in Ghaem University Hospital in Mashhad. J Paramed Sci. 2013;2(1):22-5.

45. Pourakbari B, Rezaizadeh G, Mahmoudi S, Mamishi S. Epidemiology of nosocomial infections in pediatric patients in an Iranian referral hospital. J Prev Med Hyg. 2012;53(4).

46. Soltani R, Khalili H, Abdollahi A, Rasoolinejad M, DashtiKhavidaki S. Nosocomial Gram-positive antimicrobial susceptibility pattern at a referral teaching hospital in Tehran, Iran. Future Microbiol. 2012;7(7):903-10

47. Hashemi SH, Esna AF, Tavakoli S, Mamani M. The prevalence of antibiotic resistance of enterobacteriaceae strains isolated in community-and hospital-acquired infections in teaching hospitals of Hamadan, west of Iran. J Res Health Sci. 2013;13(1):75-80

48. Heidari-soureshjani E, Heidari M, Doosti A. Epidemiology of 
urinary tract infection and antibiotic resistance pattern of E. coli in patients referred to Imam Ali hospital in Farokhshahr, Chaharmahal va Bakhtiari, Iran. J Shahrekord Univ Med Sci. 2013;15.

49. Shojaei S, Rahimi T, Amini M, Shams S. Survey of nosocomial infections in patients admitted to Nekoei hospital of Qom city in 2012, Iran. J Qom Univ Med Sci. 2015;9(4):64-73.

50. Saadat S, Solhjoo K, Norouz-nejadfard MJ, Kazemi A, Rouhi R, Mardaneh J. The frequency of Staphylococcus aureus among Shiraz hospital personnel and determination of their antibiotic sensitivity pattern. Iran South Med J.. 2014;17(5):916-26.

51. Abedini M, Ghotbi N, Hadavi N, Chavoshi D, Asgharian N. Comparison of two nosocomial infection surveillance in a neonatal ward. . Tehran Univ Med J. 2014;71(10):652-9.

52. Akhavan TF, Eslami G, Zandi H, Mousavi SM, Zarei M. Prevalence of blaVIM, blaIPM and blaNDM Metallo-Beta-Lactamases Enzymes in Pseudomonas aeruginosa Isolated from Burn Wounds in Shahid Sadoughi Burn Hospital, Yazd, Iran. J Isfahan Med Sch. 2014;31(263): $1955-64$.

53. Shakib P, Lavakhamseh H, Mohammadi B. The prevalence of nosocomial infection in ICU, Besat Hospital, Sanandaj City, Iran. Zanco J Med Sci. 2014;15(45):36-41.

54. Davoudi AR, Najafi N, Shirazi MH, Ahangarkani F. Frequency of bacterial agents isolated from patients with nosocomial infection in teaching hospitals of Mazandaran University of Medical Sciences in 2012. Caspian J Intern Med. 2014;5(4):227.

55. Makhlogi S, Salehi B, Abdi M. Frequency of nosocomial pneumonia in ICU Qazvin Razi hospital (2013). . J Qazvin Univ Med Sci. 2016;20(5):74-8.

56. Bijari B, Abbasi A, Hemati M, Karabi K. Nosocomial infections and related factors in southern khorasan hospitals. Iran J Med Microbiol. 2015;8(4):69-73.

57. Behzadnia S, Davoudi A, Rezai MS, Ahangarkani F. Nosocomial infections in pediatric population and antibiotic resistance of the causative organisms in north of iran. Iran Red Crescent Med J. 2014; 16(2).

58. Saeidimehr S, Geravandi S, Rahim F, Yosefi F, Salmanzadeh S, Foruozandeh $\mathrm{H}$, et al. Nosocomial infection rates during one year in naft grand hospital, Ahvaz, Iran. Jundishapur J Health Sci. 2015;7(4):e30124.

59. Motamedifar M, Ebrahim-Saraie HS, Mansury D, Nikokar I, Hashemizadeh Z. Prevalence of etiological agents and antimicrobial resistance patterns of bacterial meningitis in Nemazee Hospital, Shiraz, Iran. Arch Clin Infect Dis. 2015;10(2):e22703.

60. Hajibagheri K, Afrasiabian S. An epidemiologic study of nosocomial infections and its related factors at the intensive care unit of Tohid Hospital, in Sanandaj during 2003-2004. J Kurdistan Univ Med Sci. 2006;10(4):44-50

61. Hosseini F, Kargar M. Study of Frequency of Vana, Vanb and VanC1/C2 Genes in Vancomycin Resistant Enterococci Strains Isolated from Hospitalized and Non-Hospitalized Patients in South of Fars province. J Shahid Sadoughi Univ Med Sci. 2017;24(12):963-71.

62. Servatyari K, Hamzehpour H, Rasouli M. The Prevalence and Types of Burn Wound Infection in the Burn Ward of Tohid Hospital in Sanandaj in 2015: A Short Report. J Rafsanjan Univ Med Sci. 2018;16(9):883-90.

63. Rahmanian V, Shakeri H, Shakeri M, Rahmanian K, Rahimi M. Epidemiology of nosocomial infections in patients admitted to hospitals in Jahrom-2016. J Jahrom Univ Med Sci. 2017;15(1).

64. Ghafourifard M, Joolaee S, Sobhani M, Hooshmand A. The Correlation between Nosocomial Infections and Nurses' Work Environment. J Health Promo Manag. 2017;6(3):44-51.

65. Farzanpoor F, Rabiee MH, Fattahi AM. The prevalence of nosocomial infections in vasei hospital of sabzevar during. J Sabzevar Univ Med Sci. 2009-2013. 2018.

66. Dadmanesh M, Nojoomi F, Ghorban K, Allame A, Razzaghi H. A Comparison Study of Bacterial Nosocomial Infections in Two Hospitals in Tehran in 2013-2014. Paramed Sci Mil Health. 2017;12(3):23-8.

67. Heydarpour F, Rahmani Y, Heydarpour B, Asadmobini A. Nosocomial infections and antibiotic resistance pattern in open-heart surgery patients at Imam Ali Hospital in Kermanshah, Iran. GMS Hyg Infect Control. 2017;12.

68. Eshrati B, Asl HM, Afhami S, Pezeshki Z, Seifi A. Health careassociated infections in Iran: a national update for the year 2015. Am J Infect Control. 2018;46(6):663-7.
69. Ghanbari F, Ghajavand H, Behshod P, Ghanbari N, Khademi F. Prevalence of Hospital-Acquired Infections in Hospitalized Patients in Different Wards of Shariati Hospital of Isfahan, 2014. J Health. 2018;8(5):511-7.

70. Nasiri MJ, Goudarzi AM, Aslani HR, Goudarzi M, Zamani S, AdinehKharrat S. Nosocomial Infections Caused by Drug-Resistant Bacteria in a Referral University Hospital, Tehran, Iran. Nove Biomed. 2019;7(2):64-70.

71. Rahimi-Bashar F, Karami P, Khaledi A, Dehghan A, Seifrabie MA, Yaghoobi MH. Evaluation of the prevalence of nosocomial infection in different wards of Be'sat hospital of Hamedan. Avicenna Journal of Clin Microbiol Infect. 2018;5(2):31-5.

72. Kohestani SM, Rahmani H, Nourbakhsh S, Habibi F, Rajabi Vasoukolaei G. Epidemiology and Determine the Causes of Nosocomial Infectioin Teaching Hospital of Tehran: A CrossSectional Study. J Hospital. 2019;18(3):53-61.

73. Alkhudhairy MK, Saki M, Seyed-Mohammadi S, Jomehzadeh N, Khoshnood S, Moradzadeh M, et al. Integron frequency of Escherichia coli strains from patients with urinary tract infection in Southwest of Iran. J Acute Dis. 2019;8(3):113.

74. Azimi T, Maham S, Fallah F, Azimi L, Gholinejad Z. Evaluating the antimicrobial resistance patterns among major bacterial pathogens isolated from clinical specimens taken from patients in Mofid Children's Hospital, Tehran, Iran: 2013-2018. Infect Drug Resist. 2019;12:2089.

75. Piruozi A, Forouzandeh H, Farahani A, Askarpour M, Mohseni P, Fariyabi F, et al. Frequency of Nosocomial Bacterial Infections in Hospitalized Patients Referred to Amir Al-Momenin Hospital, Gerash, Iran. Genes Cells. 2019;6(3).

76. Pourkazemi A, Farashbandi H, Balu H. Epidemiological study of nosocomial infections and antibiotic resistance patterns In Guilan. Yafteh. 2019;21(1)

77. Sepandi M, Motahari F, Taheriyan M, Hashemi S. Evaluation of the Prevalence of Nosocomial Infections and their Antibiotic Resistance in one of the military hospitals affiliated Army Medical University during 2018-2019. Physic Nurs War. 2021;7(25):24-32.

78. Mansori S, Shakeri-Moghadam A, Khaledi A. Investigation of prevalence and antibiotic resistance pattern of bacteria isolated from urinary tract infections in women referred to Ghaem hospital in Mashhad. J Kashan Univ Med Sci. 2019;23(3):301-7.

79. Nejad MA, Ahmadipour M, Sohrevardi SM. Comparison of Prevalence of Nosocomial Infections in Opioid Addicts and Nonaddicts Admitted to Intensive Care Units. Int J Life Sci Pharma Res. 2020;10(2):P40-5

80. Emami A, Pirbonyeh N, Keshavarzi A, Javanmardi F, Ghermezi SM, Ghadimi T. Three Year Study of Infection Profile and Antimicrobial Resistance Pattern from Burn Patients in Southwest Iran. Infect Drug Resist. 2020;13:1499-506.

81. Allegranzi B, Nejad SB, Pittet D. The burden of healthcareassociated infection. Hand hygiene: a handbook for medical professionals 1st edition ed hospital medicine: current concepts Hoboken: Wiley. 2017:1-7.

82. Ghashghaee A, Benis MR, Aryankhesal A, Tanha K, Hosseinifard $\mathrm{H}$, Janani L, et al. The Prevalence of Hospital-Acquired Infections in the EMRO: A Systematic Review and Meta-Analysis from 2000 to 2018. Lancet Infect Dis. 2019;19(5):520-558

83. Braithwaite J, Mannion R, Matsuyama Y, Shekelle P, Whittaker S, Al-Adawi S. Health systems improvement across the globe: success stories from 60 countries: CRC Press; 2017.

84. Burke JP. Infection control-a problem for patient safety. N Eng J Med. 2003;348(7):651-6.

85. Ariyo P, Zayed B, Riese V, Anton B, Latif A, Kilpatrick C, et al. Implementation strategies to reduce surgical site infections: a systematic review. Infect Control Hosp Epidemiol. 2019;40(3):287300 . 